\title{
Retardo en la transmisión de Información en la Red Internet
}

Fausto lireire Carrera

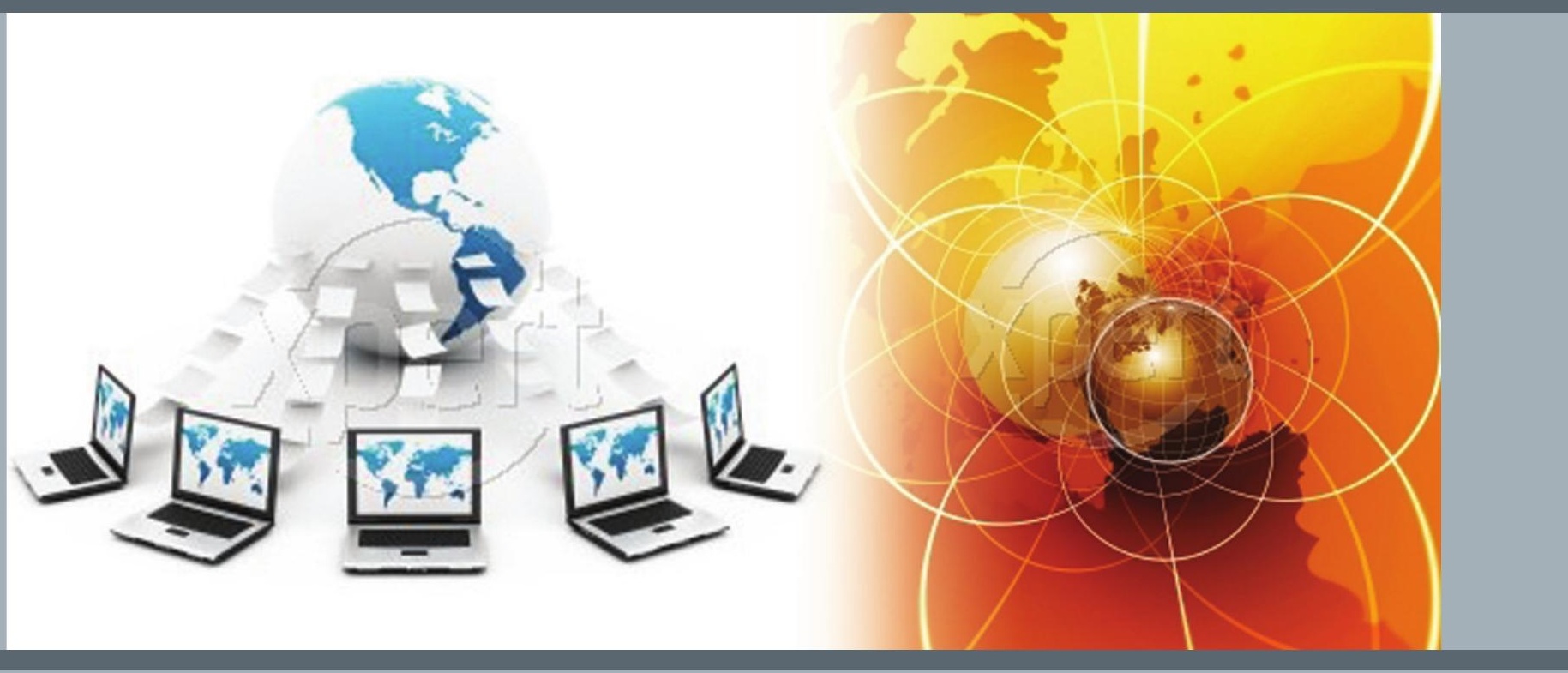

Resumen- El presente artículo trata sobre un experimento realizado para medir los retardos que sufre la información al pasar a través de la red internet desde un punto hasta otro, determinar las características de estos retardos es importante al momento de desarrollar controladores de robots controlados desde internet.

Palabras clave - Internet, control, retardo, robot móvil

El gran desarrollo tecnológico que en los últimos años ha sufrido la red Internet ha permitido ampliar su horizonte de aplicaciones, lo que ha dado lugar a la aparición de nuevas tendencias como Tele-robótica, Tele-producción, Tele-educación, Tele-cirugía, extendiéndose incluso al espacio exterior al utilizarse en los sistemas orbitales espaciales, etc.

El término "Tele-robótica" une las áreas de telemática, robótica y automatización, permitiendo al operador controlar la ejecución y control de procesos distantes, lo que conlleva con la disminución sustancial de gastos de recursos humanos y físicos.

Existen aun una serie de problemas por resolver en este campo, lo que ocupa gran parte de los recursos de investigadores por todo el mundo, porque la aplicación de la Tele-robótica es excepcionalmente importante.

1 Fausto Freire Carrera, Subdirector de la Dirección General de Posgrados, 095800212, ffreire@ute.edu.ec
Uno de estos problemas con los que nos encontramos al momento de controlar específicamente un robot móvil, cuando utilizamos la red Internet como el canal de enlace es el retardo que sufren las señales. Los protocolos TCP/IP utilizados en Internet, no garantizan que este retardo que sufren las señales durante el paso por la red sea un parámetro constante. Esto significa que el sistema de control automático del robot debe estar preparado para disminuir al mínimo los problemas que surjan por este efecto.

El valor de este parámetro de retardo de las señales en la red Internet está ligada a muchos factores, tales como:

- La velocidad de conmutación de los nodos

- La carga de los nodos

- La capacidad de tráfico de las redes

- La cantidad de trasferencia de dados

- La velocidad de la entrega, etc.

Pero los factores dominantes que determinan la magnitud del retardo principalmente son: la velocidad de la red y la carga de los nodos, por eso esta razón, esta magnitud es un valor imprevisible.

Existen varios métodos para realizar el Tele-control de los robots móviles a través de la red Internet [1], uno de estos métodos es el denominado control directo y continuo [2] y consiste en que el robot sigue las órdenes enviadas por el operador, figura 1. 


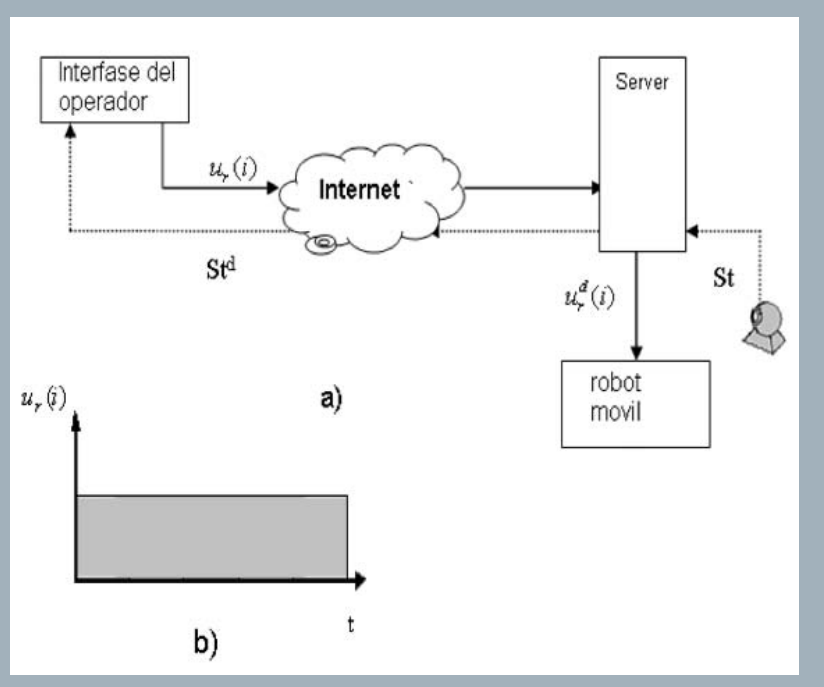

Figura 1. Análisis del Extracto Celular de la Planta (ECP) de cada uno de los tratamientos

Donde:

\begin{tabular}{|c|l|}
\hline$u_{r}^{d}(i)$ & i-mo comando que atraviesa la red Internet \\
\hline$u_{r}(i)$ & i-mo comando \\
\hline$S t^{d}$ & $\begin{array}{l}\text { flujo de la señal de video que atraviesa la red } \\
\text { Internet }\end{array}$ \\
\hline$S t$ & flujo de la señal de video \\
\hline
\end{tabular}

Este método de control es de fácil aplicación ya que toda la responsabilidad del control recae directamente sobre el operador, que es el encargado de guiar al robot, el cual controla con comandos a través de una aplicación cliente-servidor y mediante una cámara que permite obtener la información necesaria sobre la ubicación del robot.

\section{El comando Ping}

Ping es el principal comando de TCP/IP que se utiliza para solucionar problemas de conectividad, accesibilidad y resolución de nombres.

Comprueba la conectividad de nivel IP con otros equipos al enviar mensajes de solicitud de eco de ICMP (Protocolo de mensajes de control Internet).

Permite visualizar la recepción de los mensajes de solicitud de eco correspondiente, junto con sus tiempos de ida y vuelta.

\section{El protocolo ICMP}

Permite a equipos de una red compartir información de estado y errores, es el protocolo obligatorio que administra en la pila de protocolos TCP/IP, informa sobre los errores y abastece los enlaces entre los nodos de las redes. El protocolo
ICMP se utiliza por el comando Ping para el descubrimiento y la eliminación de errores de TCP/IP.

\section{El protocolo TCP/IP}

El Protocolo de control de transporte/Protocolo Internet (TCP/IP, Transport Control Protocol / Internet Protocol) es el protocolo de red más utilizado y la base de Internet que conecta ordenadores de distinta arquitectura y diferentes sistemas operativos. El protocolo TCP/IP incluye los estándares para los enlaces entre los ordenadores y el acuerdo de la unión de las redes y las reglas del enrutamiento de los mensajes.

En una red TCP/IP, tiene que proporcionar direcciones IP a los clientes. Puede que los clientes también necesiten un servicio de nombres o un método de resolución de nombres [3].

\section{Retardo en la red Internet}

Para medir el retardo que sufre las señales durante su paso por la red Internet se ha utilizado el siguiente experimento: Desde un ordenador situado en un punto A se envió señales a otro situado en un punto B, la distancia entre estos dos puntos fue de $10.000 \mathrm{Km}$. aproximadamente, estas señales se enviaron mediante la utilización del comando ping, este experimento se realizó durante siete días, en horarios diferentes, cada día se realizó 1000 mediciones en las cuales se contabilizó el tiempo que tarda la señal en ir y volver desde la fuente hasta el destino, la longitud del paquete de datos utilizado en los experimentos fue de 32 bytes.

En la siguiente tabla tenemos la lista de nodos a través de los cuales pasó la señal, cabe recalcar que el número de nodos durante el experimento prácticamente no cambio.

Tabla 1. Lista de nodos

\begin{tabular}{|c|c|}
\hline $\begin{array}{c}\text { Número } \\
\text { del nodo }\end{array}$ & $\begin{array}{c}\text { Nombre y dirección IP del } \\
\text { nodo }\end{array}$ \\
\hline 0 & pcx.localhost. [10.1.0.143] \\
\hline 1 & 10.1.0.1 (KSTU) \\
\hline 2 & runnet-gw.kursk.ru [82.179.87.137] \\
\hline 3 & m9-2-gw.runnet.ru [194.85.37.241] \\
\hline 4 & ru-msk-gw.tv11.msk.runnet.ru [194.85.40.26] \\
\hline 5 & b57-2-gw.spb.runnet.ru [194.85.40.29] \\
\hline 6 & spb-gw. RUN.Net [193.232.80.253] \\
\hline 7 & sth-gw. RUN.Net [193.232.80.206] \\
\hline 8 & s-bb1-pos7-1-0.telia.net [213.248.66.5] \\
\hline 9 & hbg-bb1-pos6-0-0.telia.net [213.248.64.30] \\
\hline 10 & adm-bb1-pos7-0-0.telia.net [213.248.65.153] \\
\hline 11 & adm-b3-pos0-0-0.telia.net [213.248.64.158] \\
\hline 12 & if-1-1.core1.AD1-Amsterdam.teleglobe.net [80.231.80.9] \\
\hline 13 & if-6-0.mcore3.LHX-London.teleglobe.net [80.231.80.30] \\
\hline 14 & if-7-0.mcore4.NJY-Newark.teleglobe.net [195.219.196.22] \\
\hline 15 &
\end{tabular}




\begin{tabular}{|c|c|}
\hline $\begin{array}{c}\text { Número } \\
\text { del nodo }\end{array}$ & $\begin{array}{c}\text { Nombre y dirección IP del } \\
\text { nodo }\end{array}$ \\
\hline 16 & if-9-0.core1.AEQ-Ashburn.teleglobe.net [216.6.63.22] \\
\hline 17 & 63.243 .149 .122 \\
\hline 18 & ae1.p420.msr2.dcn.yahoo.com [216.115.96.185] \\
\hline 19 & ge5-2.bas1-m.dcn.yahoo.com [216.109.120.151] \\
\hline 20 & alteon6.124.dcn.yahoo.com [216.109.124.8] \\
\hline 21 & a1.search.vip.dcn.yahoo.com [216.155.200.155] \\
\hline
\end{tabular}

En la figura 2 tenemos el gráfico geográfico de la ruta de la señal desde el origen hasta el destino.

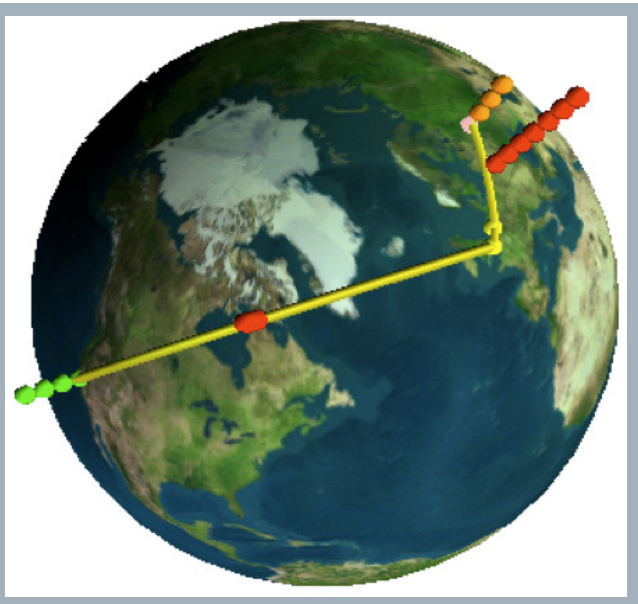

Figura 2. Ruta de la señal.

Los datos obtenidos luego de ser sometidos a análisis se presentan en forma de histograma, el mismo que permite visualizar el comportamiento de los retardos de la señal en Internet durante la semana que duró el experimento (Figura 3).

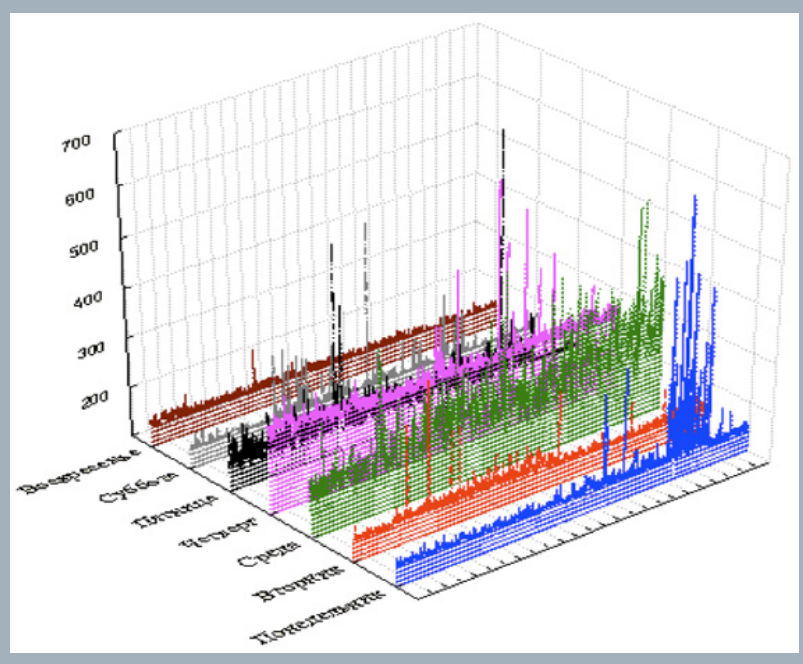

Figura 3. Histograma de los retardos de la señal en la red Internet.

En la tabla 2 se muestran los datos estadísticos obtenidos durante el experimento.

\begin{tabular}{|l|l|l|l|l|l|l|l|}
\hline & Mean & $\begin{array}{l}\text { Me- } \\
\text { dian }\end{array}$ & Min & Max & Variance & Std. Dev. & $\begin{array}{l}\text { Standard } \\
\text { Error }\end{array}$ \\
\hline Lunes & 154,6990 & 146 & 142 & 652 & 1534,245 & 39,16944 & 1,238646 \\
\hline Martes & 152,1850 & 148,5 & 142 & 403 & 251,863 & 15,87018 & 0,501859 \\
\hline Miércoles & 241,7040 & 231 & 159 & 533 & 2515,528 & 50,15504 & 1,586042 \\
\hline Jueves & 262,5280 & 260 & 238 & 602 & 500,874 & 22,38022 & 0,707725 \\
\hline Viernes & 166,6310 & 161 & 142 & 632 & 850,822 & 29,16885 & 0,922400 \\
\hline Sábado & 146,6290 & 141 & 139 & 472 & 335,423 & 18,31455 & 0,579157 \\
\hline Domingo & 146,1450 & 143 & 141 & 226 & 36,296 & 6,02464 & 0,190516 \\
\hline
\end{tabular}

Tabla 2. Datos estadísticos

A continuación se presenta el análisis diario de los resultados obtenidos, con la finalidad de tener una panorámica de las leyes de distribución a las cuales se asemejan los resultados randómicos obtenidos.

Esto es vital pues permitirá generar un modelo del comportamiento de la red Internet, que será aplicable a posteriores investigaciones, en las cuales se utilice el Internet como el medio de transmisión y recepción de información entre el operador y el robot.
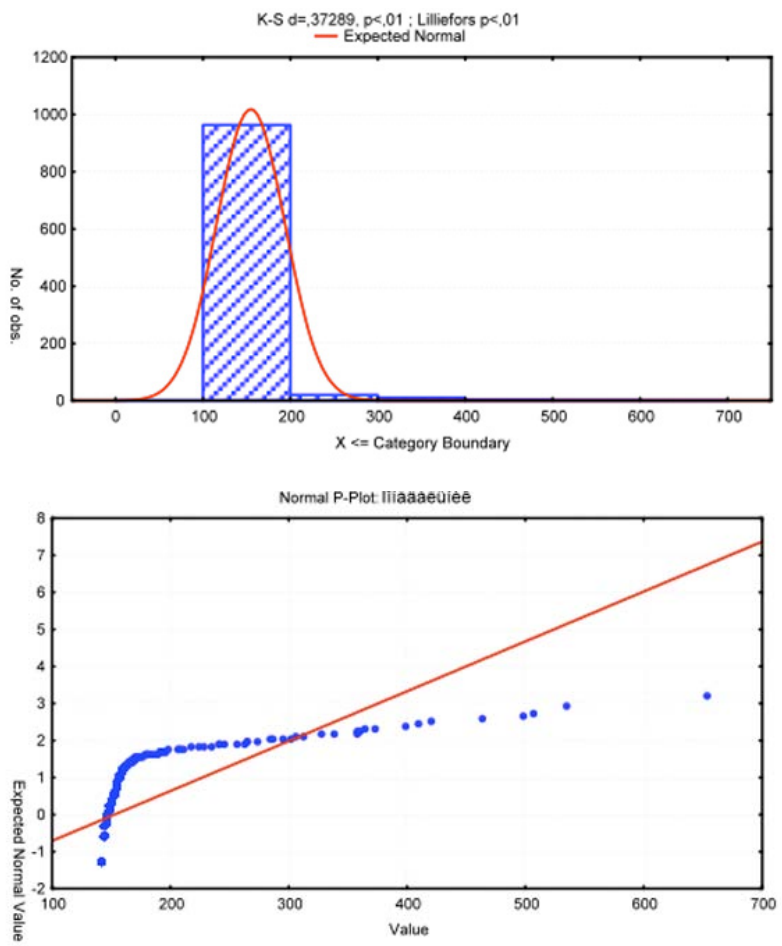

Figura 4. Histograma y Normal P-Plot del lunes

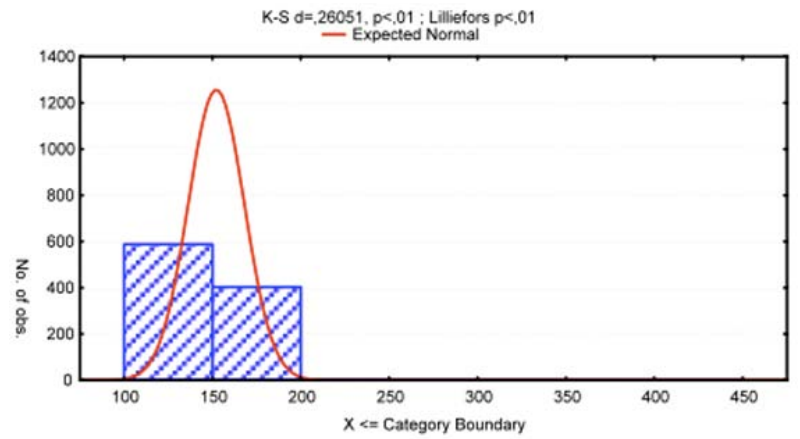




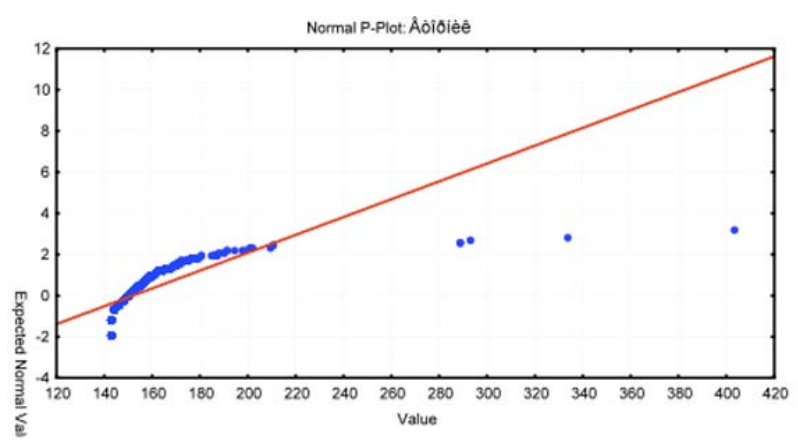

Figura 5. Histograma y Normal P-Plot del martes
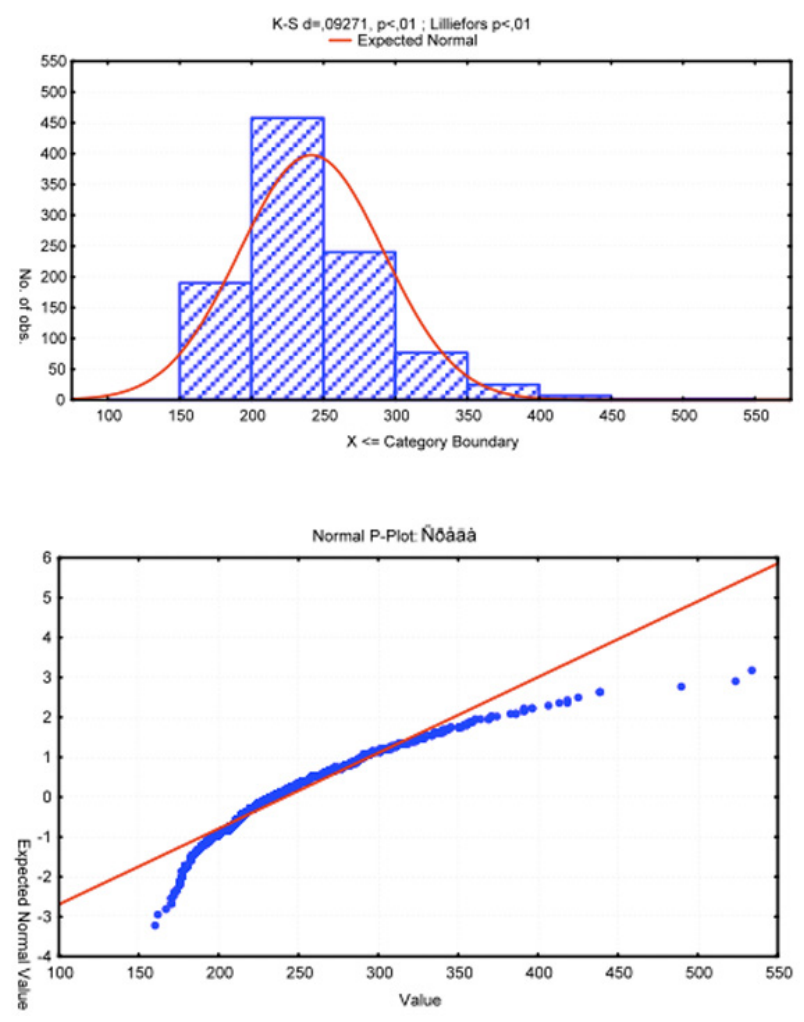

Figura 6. Histograma y Normal P-Plot del miércoles

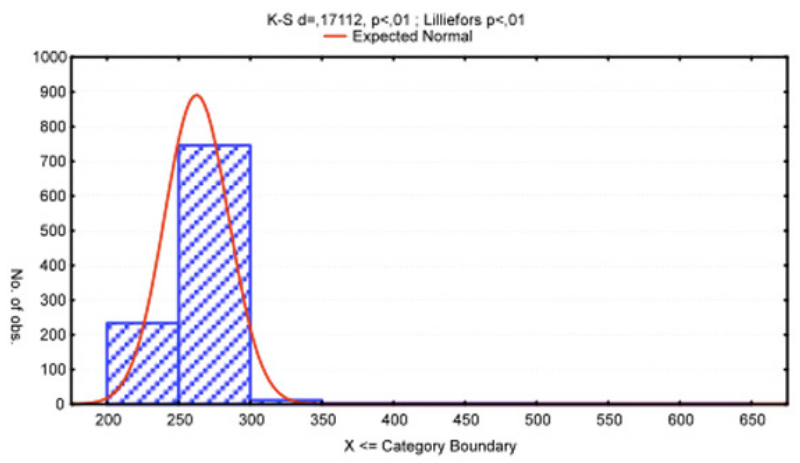

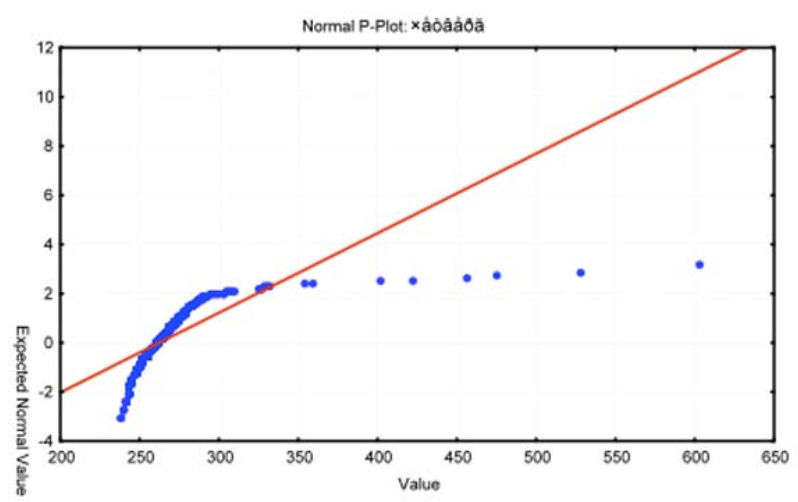

Figura 7. Histograma y Normal P-Plot del jueves
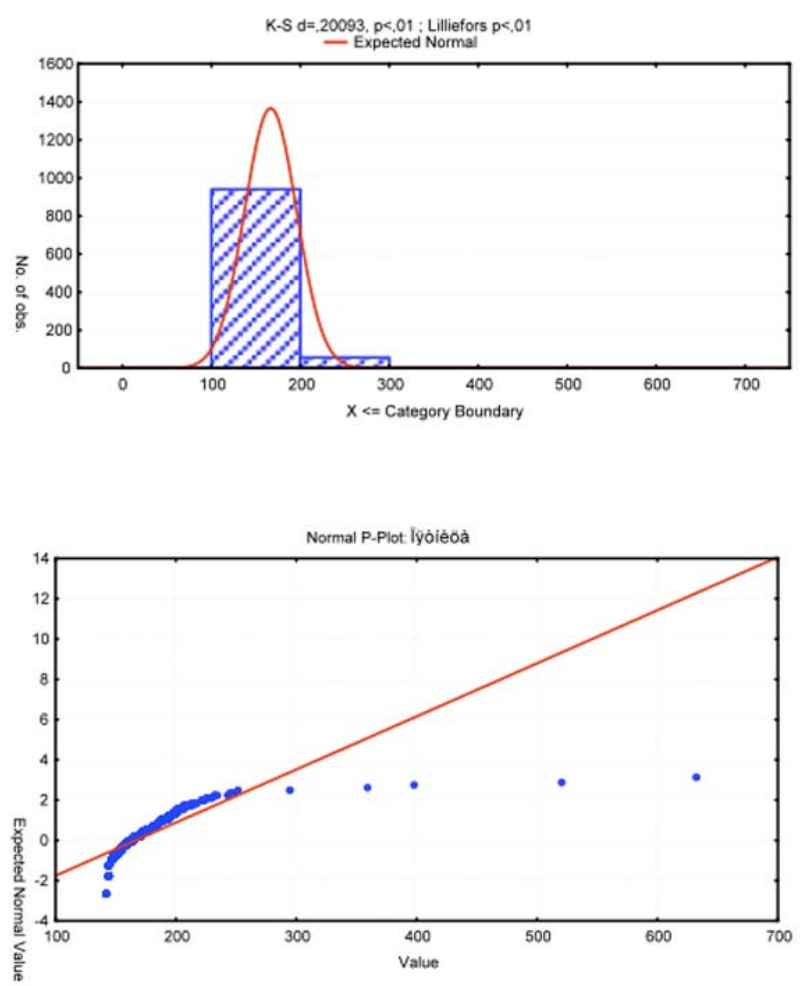

Figura 8. Histograma y Normal P-Plot del viernes

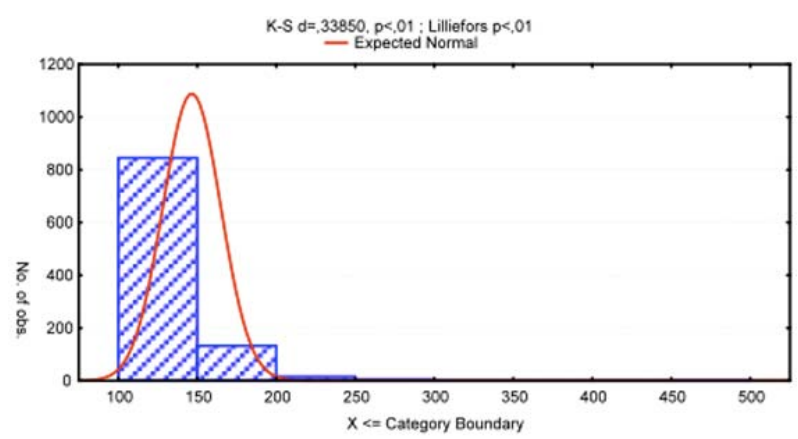




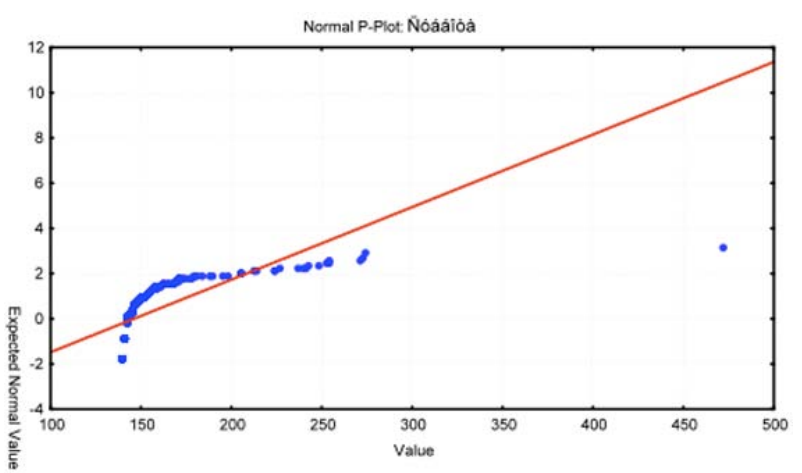

Figura 9. Histograma y Normal P-Plot del sábado
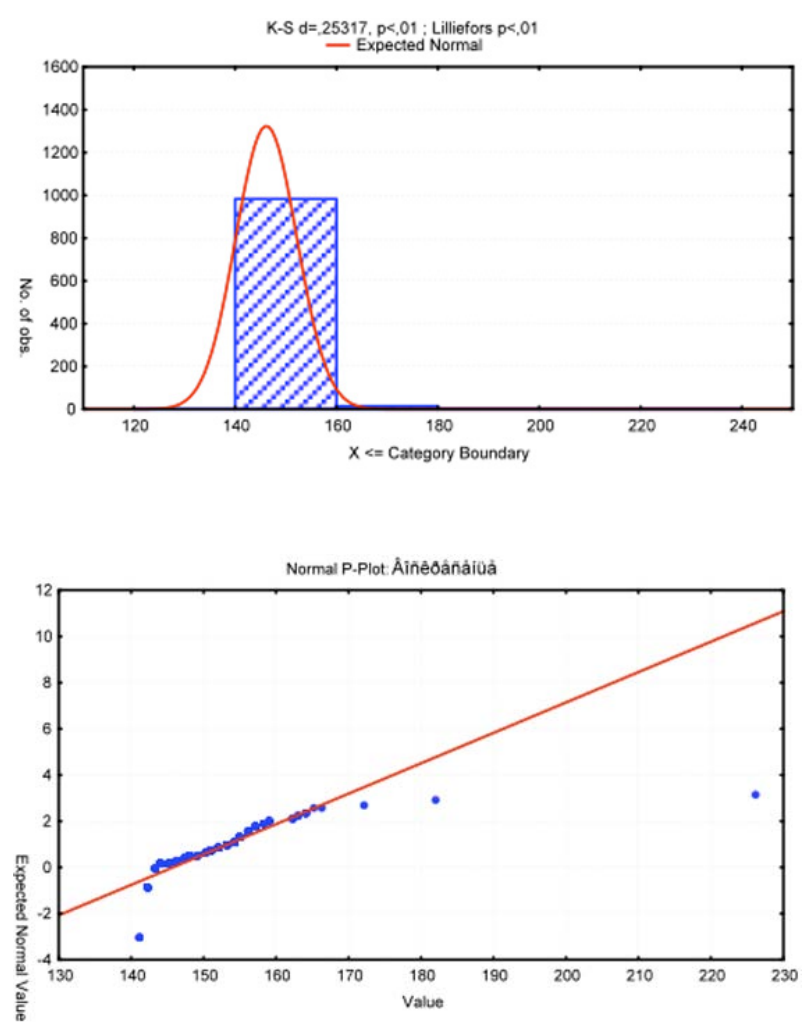

Figura 10. Histograma y Normal P-Plot del domingo

Los datos experimentales obtenidos nos llevan a la conclusión que durante la elaboración del algoritmo de control es necesario tener en cuenta todos estos retardos de la señal, ya que pueden llevar al robot a un estado de inestabilidad.

Este análisis ha permitido utilizar la ley de Distribución Normal como aceptable, para generar retardos aleatorios al momento de modelar el control del robot desde Internet.

\section{Bibliografía}

- Freire F., y otros, Control de robots móviles desde Internet, // Telecomunicaciones, Moscú, № 3, 2005, Pág.18-24.
- Lynn Conway, Richard A. Volz, and Michael W. Walker. Teleautonomous systems: Projecting and coordinating intelligent action at a distance. IEEE Transactions on Robotics and Automation, 6 (2): Pag. 146-158, April 1990.

- Jofer Procis, Manual de TCP/IP, PC Magazine, November 19, 1996, Pag. 223

- Piones H.C., Cálculo Diferencial e Integral, T.2, Press, 2001.

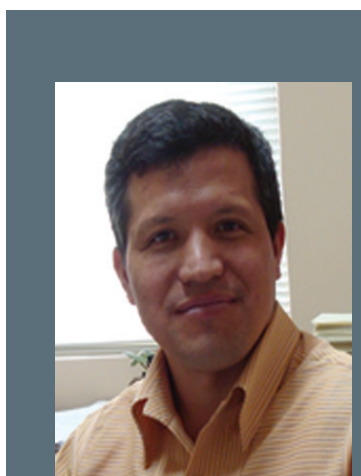

Autor: Fausto Rodrigo Freire Carrera

ffreire@ute.edu.ec

Título: Ingeniero en Sistemas.

Institución: Universidad Técnica Estatal de Kursk - UTEK (Federación de Rusia).

Facultad: Ingeniería en Sistemas, Computación y Automatización.

Título: Máster en Ciencias.

Institución: Universidad Técnica Estatal de Kursk (Federación de Rusia).

Facultad: Facultad de Ingeniería en Sistemas, Computación y Automatización.

Título: Máster en Tecnologías de la Información para la Fabricación.

Institución: Universidad Politécnica de Madrid (España).

Nombre del programa académico: Departamento de Sistemas Inteligentes Aplicados.

Título: Especialista en Robótica.

Institución: Universidad Politécnica de Madrid (España).

Nombre del programa académico: Departamento de Sistemas Inteligentes Aplicados.

Título: Ph.D en Dinámica, Resistencia, Aparatos y Dispositivos Institución: Universidad Técnica Estatal de Kursk (Federación de Rusia).

Nombre del programa académico: Facultad de Mecánica Teórica y Mecatrónica. 